Thorax (1976), 31, 309.

\title{
Long-term beclomethasone dipropionate aerosol therapy in juvenile asthma
}

\author{
R. S. F R A N C IS \\ Whipps Cross Hospital and Chest Clinic, London E11
}

\begin{abstract}
Francis, R. S. (1976). Thorax, 31, 309-314. Long-term beclomethasone dipropionate aerosol therapy in juvenile asthma. Following a short-term clinical trial reported elsewhere, beclomethasone dipropionate aerosol has been given to 15 children with asthma for between $2 \frac{1}{2}$ and 3 years except for a short placebo period after the first year. Month-bymonth records of wheezing, peak flow rate, and other treatments used are presented for the first 17 months, adrenocortical function tests are reported for the first 2 years, and growth is recorded for $2 \frac{1}{2}-3$ years. The short-term clinical benefits of the treatment are confirmed in the longer term, adrenocortical function appears to be unchanged, and growth proceeds along expected lines. The main disadvantage seems to be worsening of eczema and allergic rhinitis in those children who have ceased using corticotrophin or oral steroids for the control of asthma. It is concluded that in the long term beclomethasone dipropionate aerosol provides safe and effective day-to-day control of asthma in children, although occasional recourse to systemic steroid therapy cannot be avoided. Oral candidiasis has not been a clinical problem.
\end{abstract}

In 1972 a short trial of beclomethasone dipropionate (BDP) aerosol was undertaken in 37 asthmatics, of whom 15 were children aged 6 to 17 , with whom this paper is concerned.

Children were included in the trial if they had severe asthma-defined as airways obstruction varying widely in severity over short periods of time either spontaneously or as a result of treatment-for the control of which steroid therapy had been or was to be instituted. The group comprised 12 boys and 3 girls of mean age 10.7 years. Because of their asthma, all had regular disturbance of sleep and frequent absence from school and were seriously disadvantaged in games. All had perennial symptoms, 6 having seasonal exacerbations. Eczema was present in 10, allergic rhinitis in 9 , and 11 had a family history of allergic rhinitis, asthma, or both. Asthma had been present for from 2 to 14 years (mean duration 7.4 years), and most had been under observation for at least one year. Twelve had received regular steroid support (although only 9 were being so treated in the month immediately before starting BDP). In the remaining three cases, steroid therapy was in immediate prospect.

Before the trial the children were established on treatment regimens of disodium cromoglycate and salbutamol aerosol in addition to corticotro- phin or prednisolone. They-or their parentswere well trained in self-medication and were free to select their own day-to-day treatment, recording all doses in a daily diary together with a simple letter code to indicate the severity of their wheezing $(\mathrm{A}=$ none, $\mathrm{B}=$ slight, $\mathrm{C}=$ troublesome, and $\mathrm{D}=$ severe). Each week at the clinic records were made of height, weight, peak flow rate (PFR), and forced expiratory volume at 1 second $\left(\mathrm{FEV}_{1}\right)$. After a control period of one month, treatment was started with BDP aerosol, $300 \mu \mathrm{g}$ daily (6 puffs of $50 \mu \mathrm{g}$ ), and continued for eight weeks. Thirty-minute tetracosactrin tests of adrenocortical function were performed at the end of control and trial periods. The results showed that, compared with treatment in the control period, BDP promoted significant improvement in wheezing, PFR, and FEV , and led to a marked reduction in the use of other medications without impairing adrenocortical function (Francis, 1975). These findings confirmed other reports of the value of BDP aerosol in juvenile asthma (Brown and Storey, 1973; Dickson et al., 1973; Godfrey and Konig, 1973).

\section{METHODS OF PRESENT STUDY}

Following this initial study, beclomethasone therapy has been continued on a long-term basis, 
and the children's progress has been monitored in respect of growth, ventilatory performance, symptoms, daily medication, and adrenocortical function for a total period of 30-36 months. On the first anniversary of the original 8-week trial, a placebo was substituted for the active aerosol and all procedures of the original trial were repeated. It was proposed to continue the placebo experiment for 8 weeks but this was not achieved in all cases. The adrenocortical function test was repeated on withdrawing the placebo aerosol and active aerosols were then restored. In the present report of month by month progress of the $1 \overline{1}$ children (see Fig. 1), all values are reported as means for the whole group each month. Thus? wheezing is expressed as the mean percentage os days each month when it was absent (code A) of else troublesome or severe (codes $\mathrm{C}$ or $\mathrm{D}$ ). Peak flow is expressed as

$$
\frac{\text { observed PFR }}{\text { predicted PFR }} \times 100,
$$

using data by Cotes (1965), and allowing foím growth each month. Accounts of clinical trials ito

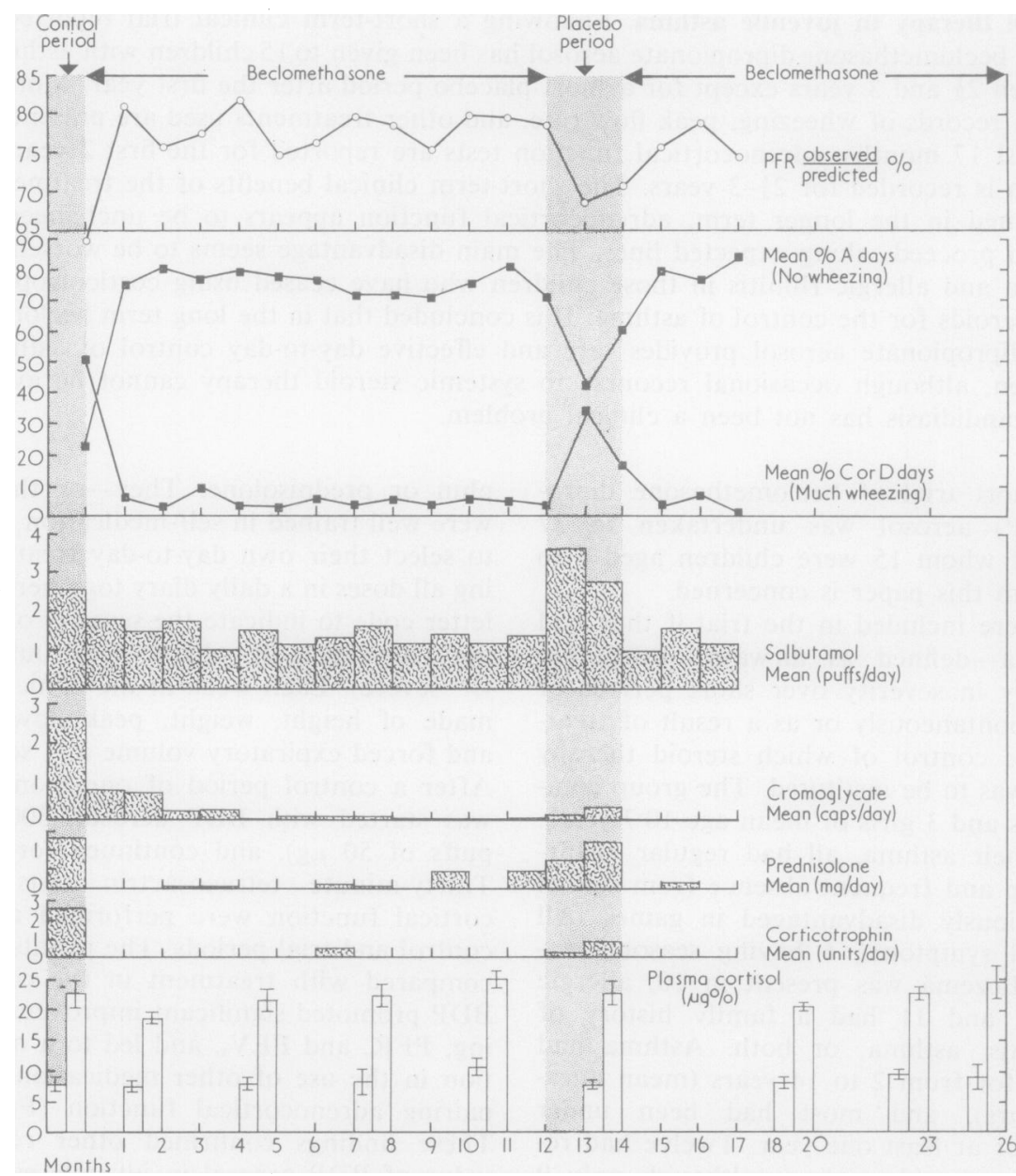

FIG. 1. The figure summarizes the collective observations on 15 children during an initial control period of 1 month, followed by 12 months on BDP aerosol. After a period of 2 months on placebo aerosol, BDP treatment is resumed. The units adopted are explained in the text. Monthly values are means for all 15 children. Tetracosactrin tests are shown as double columns representing mean values for resting and stimulated plasma cortisol, with standard errors shown as bars. 
asthma which omit details of other antispasmodic treatments have been justly criticized by Herxheimer (1972), and for this reason all medication used is shown as the mean daily dosage for all children per month. Adrenocortical function test results are shown as paired columns representing resting and stimulated plasma cortisol levels. These tests were performed before starting BDP, at 3-monthly intervals afterwards in the first year, and thereafter at approximately 20,23 , and 26 months. The growth of each child has been plotted on height and weight standard charts for

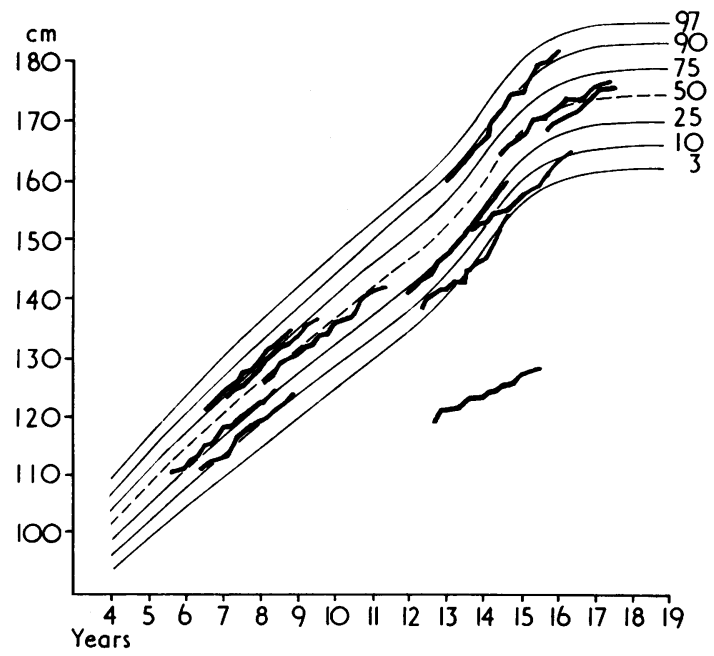

FIG. 2. Height centiles. Asthmatic boys on beclomethasone dipropionate aerosol.

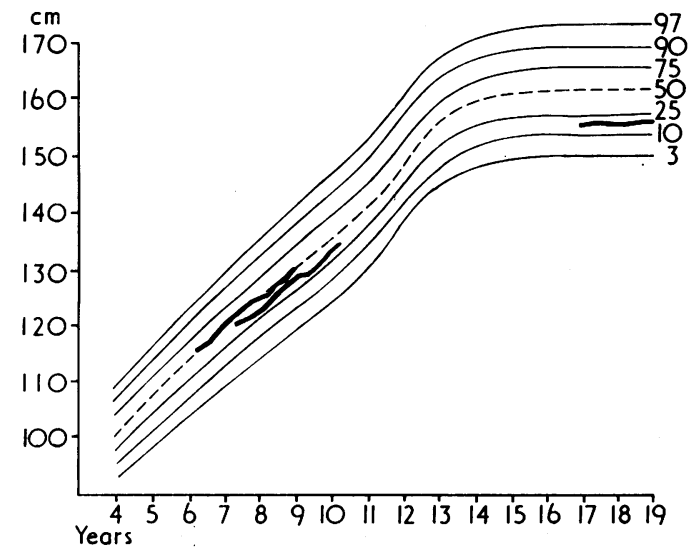

FIG. 3. Height centiles. Asthmatic girls on beclomethasone dipropionate aerosol.

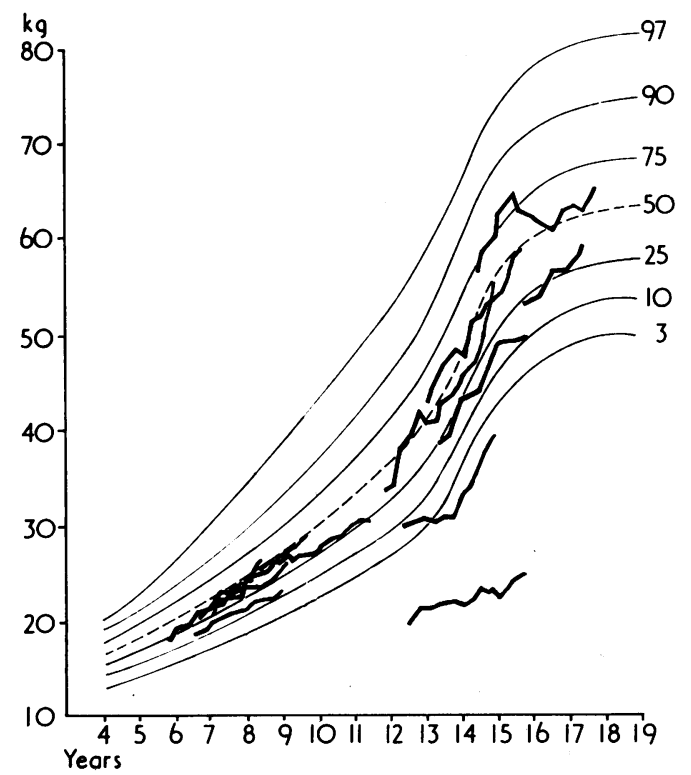

FIG. 4. Weight centiles. Asthmatic boys on beclomethasone dipropionate aerosol.

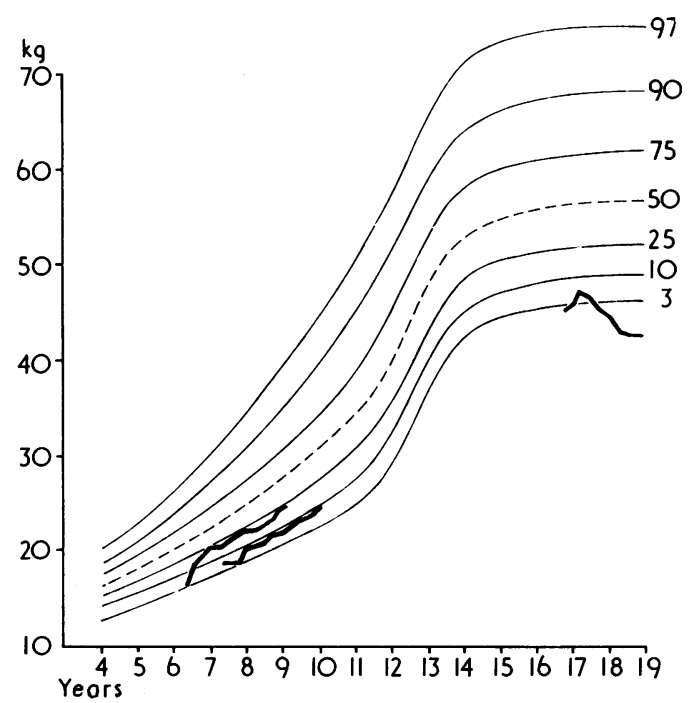

FIG. 5. Weight centiles. Asthmatic girls on beclomethasone dipropionate aerosol.

$2 \frac{1}{2}-3$ years (Figs 2-5). After the first year regular records were kept of oral symptoms or signs which might be attributed to the use of BDP aerosol, and routine swabs and cultures for Candida were examined. 


\section{RESULTS}

MONTHS 0-2 (initial trial) Data for the control month preceding the use of BDP show that, despite the use of salbutamol aerosol, cromoglycate, corticotrophin and, to a lesser extent, prednisolone, control of symptoms was indifferent. Only $22 \%$ of days were free of wheezing (code A) and on $51 \%$ it was troublesome or severe (codes C or D). The first month on BDP showed an abrupt improvement, A days trebling to $74 \%$, and $\mathrm{C}$ or $\mathrm{D}$ days falling to well below $10 \%$ while observed/predicted PFR\% rose from 63.8 to 81 . Statistical analysis after the first 2 months on BDP showed that these improvements were significant $(P<0.05)$. At the same time the mean daily dosage of other treatments fell, salbutamol by onethird and cromoglycate by three-quarters, while prednisolone and corticotrophin were virtually abandoned. A slight fall in mean plasma cortisol values, both resting and after stimulation, was probably due to the removal of corticotrophin stimulus in the seven patients so treated during the control period.

MONTHS 3-12 During the remainder of the first year on BDP the initial improvement was maintained, as measured by mean wheezing levels and PFR observations. Consumption of salbutamol aerosol settled at a mean of slightly more than 1 puff daily while that of cromoglycate virtually ceased after eight months. There was only one admission to hospital for asthma, this being the only single occasion when corticotrophin was used during the first year of BDP. Prednisolone was used by two children during this period, for four short periods in all.

PLACEBo PERIOD After one year on BDP, 14 children were given a placebo aerosol. Two rejected it after one and three weeks respectively; 12 used it for one month and eight for two months. Thirteen preferred the active aerosol and nine demanded a return to it. None preferred the placebo. Figure 1 shows the general effect on symptoms, PFR, use of other medications, and adrenocortical function. An abrupt reduction of A days from a mean value of $71 \%$ to $42 \%$ was accompanied by a rise of $C$ and $D$ days from $3 \%$ to $35 \%$; and mean observed $/$ predicted PFR $\%$ fell from 79 to 69 , all despite the increased use of salbutamol aerosol, prednisolone, and corticotrophin. The apparent improvement during the second placebo month merely reflects the abandonment of the inert aerosol by those whose asthma became intolerable, and the mitigating effect of increasing other treatments. Little change in adrenocortical function was noted at the en of the placebo period.

The Table records, for individual children changes in symptoms, mean PFR and other medications as a result of changing from the active to the placebo aerosol and back again. clear advantage to the active aerosol is noted. Si⿺ children used prednisolone during the placeb $\vec{\Phi}$ period compared with two during the whole of the preceding year on BDP. Two children used co $\overrightarrow{E_{j}}$ ticotrophin during the placebo period compared with one during the previous year.

T A B L E

SUBSTITUTION OF PLACEBO FOR ACTIVE AEROSOL Iब 14 CHILDREN

\begin{tabular}{|c|c|c|c|c|}
\hline & \multicolumn{3}{|c|}{ No. of Patients Showing: } & (0) \\
\hline & Increase & Decrease & No Chan & nణ \\
\hline $\begin{array}{l}\text { Change from BDP to placebo: } \\
\% \text { A days } \\
\% \text { C + D days } \\
\text { Mean PFR } \\
\text { Salbutamol use } \\
\text { Prednisolone use } \\
\text { Cromoglycate use }\end{array}$ & $\begin{array}{r}3 \\
11 \\
5 \\
11 \\
6 \\
2\end{array}$ & $\begin{array}{r}10 \\
1 \\
9 \\
1 \\
0 \\
0\end{array}$ & $\begin{array}{r}1 \\
2 \\
0 \\
2 \\
8 \\
12\end{array}$ & 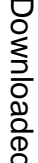 \\
\hline $\begin{array}{l}\text { Change trom placebo to BDP: } \\
\% \text { A days } \\
\% \text { C + D days } \\
\text { Mean PFR } \\
\text { Salbutamol use } \\
\text { Prednisolone use } \\
\text { Cromoglycate use }\end{array}$ & $\begin{array}{r}11 \\
0 \\
9 \\
0 \\
0 \\
0\end{array}$ & $\begin{array}{r}1 \\
10 \\
4 \\
10 \\
6 \\
2\end{array}$ & $\begin{array}{r}2 \\
4 \\
1 \\
4 \\
8 \\
12\end{array}$ & $\begin{array}{l}\overline{\overline{0}} \\
\frac{3}{3} \\
\frac{2}{\vec{Z}} \\
\overline{0}\end{array}$ \\
\hline
\end{tabular}

FinAl (POST-PLACEBO) PERIOD In Fig. 1, month marked 15,16 , and 17 represent the first three months after restoration of BDP aerosol follow ing the period on placebo. They show an immediate and sustained return to the improved clinical state noted when BDP was first started over a year previously. Detailed observation of PFR, wheezing, and all medications used has beed continued in these children for a total of $2 \frac{1}{2}-3$. years, during which time optimal control of asthma has been maintained at the level shown in Figure 1. It has occasionally been possible to reduce the dose of BDP in some cases to $100 \mu \mathrm{g}$ or even $50 \mu \mathrm{g}$ daily for weeks at a time. Adreno: cortical function tests are reported for up to 29 months and growth rates for the whole period of $2 \frac{1}{2}-3$ years (Figs $2-5$ ).

ADRENOCORTICAL FUNCTION The results of the hour tetracosactrin stimulation tests, recorded if Fig. 1 as mean plasma cortisols with the standard errors, show no appreciable change us to some 26 months after starting BDP. Not af children could be tested at each stage, the num 
bers being $14,15,15,13,13,13,11,9$, and 9 in the successive tests shown.

GROWTH Composite height and weight standard charts (Tanner, 1958) for 12 boys and three girls are presented in Figs $2-5$, covering $2 \frac{1}{2}-3$ years. These show that both sexes have grown according to expectation during the period on BDP therapy, except for one boy who stands out as well below the 3rd centile. He was born in Pakistan of short parents, his bone age is some years below the stated age, his sexual development has not started, and on testing he appears to have normal growth hormone, so there is some doubt whether his stated birth date (9 July, 1959) is correct. His growth corresponds approximately to that of a child some four years younger in the 3rd centile. Before starting BDP he had been treated with corticotrophin, during which time the slope of his growth curve had been about the same.

SIDE-EFFECTS Eczema and allergic rhinitis became more troublesome in asthmatic children weaned off corticotrophin or prednisolone but otherwise side-effects were minimal. Only one child had symptoms corresponding to the candidiasis sometimes seen in adults on BDP aerosol, viz., recurrent hoarse voice, occasional white spots in the mouth, and a smooth linear patch along the central dorsal area of the tongue. Regular buccal swabs of this child produced only one positive culture for Candida and no evidence of yeasts on direct smear. Two other children had occasional smooth oval patches on the tongue, and another had a smooth linear patch along the central dorsal area of the tongue-all asymptomatic. One child had a small tongue ulcer on BDP, and another had a similar ulcer on placebo. Routine stained buccal smears were almost uniformly negative for yeasts, and about $10 \%$ of all cultures yielded a slight or moderate growth of Candida.

\section{DISCUSSION}

Reported experience of BDP aerosol in children is mainly confined to short-term studies. Dickson and others (1973), Francis (1975), Godfrey and Konig (1973), Smith (1973), and Brown and Storey (1973) all confirm its clinical effectiveness in controlling asthma and its capacity to displace routine oral steroids or corticotrophin therapy in most cases. In a second report-and the longest follow-up study to date-Godfrey and Konig (1974) showed that benefit is continued for between 13 and 20 months; that growth and adreno- cortical function ( $1 \mathrm{mg}$ tetracosactrin, $2 \mathrm{hr}$ test) proceed normally during the first year; and that moniliasis and respiratory infections have caused no problems. The present report is an account of the collective experience of 15 asthmatic children on BDP aerosol therapy for 30-36 months, starting with a baseline period of one month on conventional treatment. The significant advantage for BDP which was apparent at an early stage was sustained throughout the first year of treatment, during which corticotrophin was used once and oral steroids four times. After a dramatic interruption of progress during substitution by an inert aerosol, clinical benefit from BDP aerosol has continued through the whole period of $30-36$ months.

There are several reasons for substituting an inert aerosol at one year. First, the original trial not being of double-blind crossover type, it seemed important to rule out the placebo effect of a new treatment, which may last up to 12 weeks, according to Silverman and others (1972). Secondly, it would tend to eliminate purely seasonal factors as a cause of the initial improvement. Thirdly, it would check whether some children had in fact 'grown-out' of their asthma during the successful year on BDP, and, finally, it would provide a valuable comparison with the initial control period before BDP was started. The results of using the inert aerosol leave no doubt that the continued improvement during the first year was in fact due to the regular use of BDP, an observation borne out by the occasional discontinuation of treatment due to carelessness or over-confidence, which was usually followed by relapse after 7-10 days. The increase in wheezing, the fall in PFR, and the increased consumption of other medication on the inert placebo recalled conditions during the initial control period 13 months previously except that, under conditions where patients (or parents) were trained in self-medication, there was hardly any recourse to DSCG, which seems therefore to be the drug with least popular appeal among the patients. Children increased their use of salbutamol aerosol during the placebo period to levels higher than those not only of the trial, but also of the control period. The preference for prednisolone rather than corticotrophin for relapse during the placebo period reflected dislike of injections. Thus, in the initial control period, there were seven patients on corticotrophin and two on prednisolone, whereas in the placebo period the ratio was reversed at two and six respectively.

In a long-term study of this type, the strict 
control of all variables is consistent with neither ethics nor patient rapport; results have to be judged by several criteria; thus a fall in PFR has more significance if accompanied by an increase in wheezing and the increased use of other medications. For this reason the findings shown in Fig. 1 have to be taken as a whole. The most dramatic changes are in wheezing scores, and while the consumption of other drugs provides good objective evidence of the clinical state, the variability of PFR provides support for the view (Chai and others, 1968; Godfrey, 1974) that these tests are most informative when recorded several times each day. The safety of this form of treatment over a period of $2 \frac{1}{2}-3$ years is confirmed by the absence of any suppressive effect on adrenocortical function, together with evidence of unhindered growth and the absence of any untoward incidence of local side-effects such as respiratory candidiasis. The data on growth, showing steady progress along the original percentile, do not suggest any acceleration after starting BDP aerosol-at least in these children, whose growth had not earlier been stunted by prolonged corticosteroid therapy. The significance of occasional smooth patches on the tongue remains to be ascertained but the possibilities of long-term mucosal atrophy must be borne in mind. The main identifiable problem resulting from BDP aerosol therapy is the worsening of nasal symptoms and eczema in those children in whom asthma control no longer calls for regular corticotrophin or oral steroid therapy, a feature noted in other reports, and one which now seems to persist with the passage of time.

The children's plasma cortisol estimations were carried out at the Hospital for Sick Children, London, and I am indebted to Professor Barbara E. Clayton for her help and advice; also to Christine Bridges for secretarial help; and to my nursing and medical colleagues, and to all the patients for their willing co-operation. Dr. David Harris, of Messrs. Allen \& Hanburys, gave much constructive help and kindly supplied the beclomethasone dipropionate aerosols.

\section{REFERENCES}

Brown, H. M. and Storey, G. (1973). Beclomethasone⿳亠丷厂 dipropionate steroid aerosol in treatment of per $\overline{6}$ ennial allergic asthma in children. British Medica $\vec{\nabla}$ Journal, 3, 161.

Chai, H., Purcell, K., Brady, K., and Falliers, C. Jes (1968). Therapeutic and investigational evaluation of asthmatic children. Journal of Allergy, 41, 23:-

Cotes, J. E. (1965). Expiratory peak flow rate (PFR in normal boys and girls. In his Lung Function, p. 319. Blackwell, Oxford.

Dickson, W., Hall, C. E., Ellis, E., and Horrocks $\stackrel{\times}{\omega}$ R. H. (1973). Beclomethasone dipropionateaerosol in childhood asthma. Archives of Disease in Childhood, 48, 671.

Francis, R. S. (1975). Beclomethasone dipropionate aerosol in adult and juvenile asthma. Frontiers of Internal Medicine, 1974 12th Int. Congr. InternalMed., Tel Aviv, 1974, pp. 236-241. Karger, Basel.c

Godfrey, S. (1974). Problems peculiar to the diagnosis and management of asthma in children. $B T T A$ Review, 4, 1 (Supplement to Tubercle, 55.) and Konig, P. (1973). Beclomethasone aerosop in childhood asthma. Archives of Disease in Child hood, 48, 665.

and - (1974). Treatment of childhood asthma for 13 months and longer with beclomethasong్ dipropionate aerosol. Archives of Disease ing Childhood, 49, 591.

Herxheimer, H. (1972). Beclomethasone aerosol in $\overrightarrow{5}$ asthma. Lancet (Correspondence). 2, 91.

Silverman, M., Connolly, N. M., Balfour-Lynn, L. $\bar{?}$ and Godfrey, S. (1972). A long-term trial of disodium cromoglycate and isoprenaline in children with asthma. British Medical Journal: 3, 378 .

Smith, J. M. (1973). A clinical trial of beclometha ج্ج sone dipropionate aerosol in children and adolescents with asthma. Clinical Allergy, 3, 249?

Tanner, J. M. (1958). The evaluation of physicaB growth and development. In Modern Trends in Paediatrics (Second Series), edited by A. Holzeb and J. P. M. Tizard, p. 325, Butterworth, London?

Requests for reprints to: Dr. R. S. Francis, Whipps Cross Hospital and Chest Clinic, London E11. 